\title{
CHARACTERISTICS OF COGNITIVE STATUS OF EPILEPSY PATIENTS TREATED WITH MONOTHERAPY OF FIRST-GENERATION ANTIEPILEPTIC DRUGS
}

\author{
Herpan Syafii Harahap ${ }^{1}$, Mohammad Rizki ${ }^{1}$, Muhammad Fabian Nurfadia ${ }^{1}$, Amalina Syadza Nabiela ${ }^{1}$ and \\ Intan Purnama Sari ${ }^{1}$
}

${ }^{1}$ Faculty of Medicine Universitas Mataram, Mataram, Nusa Tenggara Barat, Indonesia

Corresponding author:Herpan Syafii Harahap

Email: herpanharahap@unram.ac.id

\begin{abstract}
Epilepsy is one of the main health problems in neurology that can lead to cognitive decline. Generally, the epilepsyassociated cognitive decline is influenced by demographic, clinical, and treatment characteristics. This study aimed to determine the characteristics of cognitive status of epilepsy patients who received monotherapy using first-generation antiepileptic drugs (AEDs), namely phenytoin, carbamazepine, and valproic acid. It involved 93 epilepsy patients of Mutiara Sukma Mental Hospital ( $n=38)$ and Mataram General Hospital ( $n=55)$. Besides, 93 healthy patients were assigned as healthy control group $(H C)$ subjects $(n=93)$. Demographic characteristics collected from epilepsy and $H C$ groups were age, gender, and years of education. Clinical characteristics taken from both groups were MoCA-Ina score. Clinical characteristics taken from epilepsy group were age at epilepsy onset, type of seizure (partial vs generalized), etiology (idiopathic vs structural), first-generation AED used, years of treatment, and cognitive status. The result of the study revealed that there were no significant different between the two groups in the means of age and years of education as well as the frequency of male gender $(p>0.05)$. The mean of MoCA-Ina score of epilepsy group was significantly lower compared with HC group ( $p<0.05)$. The frequency of cognitive decline among epilepsy patients was $75.3 \%$. The cognitive functions of epilepsy patients using monotherapy with carbamazepine, phenytoin, or valproic acid was significantly lower compared with healthy subjects. We conclude that there was high prevalence of epilepsy-associated cognitive impairment which was associated with male gender.
\end{abstract}

Keywords: Epilepsy-associated cognitive decline, Monotherapy, First-generation AEDs, MoCA-Ina score

\section{INTRODUCTION}

Epilepsy is one of the main health problems in neurology. The overall prevalence of epilepsy in the world ranges from 0.64 to $0.76 \% 1,2$, while in Asia is about $0.15-1.4 \%^{3}$. The wide range of reported prevalence of epilepsy in Asia is influenced by the demographic and clinical characteristics of the population of epilepsy patients in different countries as well as the research methods used ${ }^{4}$. At present, the data regarding the prevalence of epilepsy in Indonesia remain scarce.

Epilepsy can lead to cognitive decline that decreases the quality of life of the patients ${ }^{5}$. It may affect one or more cognitive domains, especially attention, memory, and executive function ${ }^{6}$. Since epilepsy-associated cognitive decline can be found in new-onset epilepsy patients who have never received treatment, it is important to initially assess cognitive status of patients with epilepsy ${ }^{7}$. Early assessment and intervention slow down the progression of epilepsy-associated cognitive decline and maintain the functional capacity as well as the social function ${ }^{8,9}$. Nevertheless, this health problem as well as epilepsy per se have not been considered a priority by the health authorities in developing countries ${ }^{10}$.
The susceptibility of epilepsy patients who suffer from epilepsy-associated cognitive decline varies greatly. It is determined by demographic characteristics of the patients (age, gender, and level of education), clinical characteristics of the epilepsy (type of seizure, age at epilepsy onset, and etiology), and characteristics of the treatment (types and combination of antiepileptic drugs used $)^{11}$. In addition, it is also reported that ethnicity is deemed to be another demographic characteristic that influences the susceptibility of cognitive impairment in epilepsy patients ${ }^{12}$.

Epilepsy-associated cognitive decline occurs as a result of neuronal damage in particular brain areas that serve cognitive functions, such as hippocampus and frontal lobes, both during epileptic seizures and interictal epileptiform discharge ${ }^{13,14}$. Thus, complete elimination of epileptic seizures and interictal epileptiform discharge with antiepileptic drugs (AEDs) is very important in the management of both epilepsy and epilepsy-associated cognitive decline. On the other hand, AEDs can also have a negative impact on cognitive status, theoretically through their ability to modulate neuronal excitability ${ }^{15}$. The susceptibility of epilepsy patients to AEDsassociated cognitive decline is considered to be higher in the use of first-generation AEDs, such as 
benzodiazepine, phenobarbital, carbamazepine, phenytoin, and valproic acid.

Previous studies on the impact of first-generation AEDs on cognitive function were inconclusive. Some studies showed negative impact on cognitive function, while others reported contrasting findings ${ }^{16,17}$. At present, carbamazepine, phenytoin, and valproic acid are still commonly used, especially in developing countries, including Indonesia ${ }^{18,19}$. Some considerations for the use of these three drugs include their clear profile of efficacy, safety and side effects as well as their affordable costs compared to the new-generation AEDs ${ }^{20}$. Generally, vulnerability to the decline of cognitive status related to the use of AEDs can be reduced by using monotherapy ${ }^{15}$.

The present study aimed to determine the characteristics of cognitive status focusing on epilepsy outpatients given monotherapy of phenytoin, carbamazepine, and valproic acid. This was also the first study conducted on a subpopulation of epilepsy outpatient living in rural areas in Indonesia. Determination of the characteristics of cognitive status of patients on monotherapy of first-generation AEDs and ethnicity is important for local health authority to assess the urgency of the management of cognitive decline in these epilepsy patients.

\section{METHODS}

\section{Subjects}

This study involved epilepsy patients as well as healthy controls living in Mataram, Nusa Tenggara Barat. The present study employed an unpaired categorical analytical case-control study, hence the sample calculation formula $\left(\mathrm{n}_{1}=\mathrm{n}_{2}=\left\{\left(\mathrm{Z}_{\mathrm{a}} \sqrt{2} \mathrm{PQ}+\right.\right.\right.$ $\left.\left.\left.\mathrm{Z}_{B} \int\left(\mathrm{P}_{1} \mathrm{Q}_{1}+\mathrm{P}_{2} \mathrm{Q}_{2}\right)\right):\left(\mathrm{P}_{1}-\mathrm{P}_{2}\right)\right\}^{2}\right)$ was applied to determine the sample size. The lowest prevalence of cognitive impairment in epilepsy patients $\left(P_{2}\right)$ was about $30 \%{ }^{21}$. If $a=0.05, Z_{\alpha}=1.96, B=0.2$, $Z_{B}=0.84, P_{2}=0.3, P_{1}-P_{2}=0.2, P=\left(P_{1}+P_{2}\right): 2$, and $Q=1$ - $P$, the sample size required for each group was about 93 using the sample calculation formula mentioned above. The epilepsy patients were obtained consecutively from two referral hospitals in Mataram, i.e. Mutiara Sukma Mental Hospital and Mataram General Hospital. The inclusion criteria for this group were epilepsy patients aged 18-50 years old, at least junior high school graduate (nine-year-education), and received AEDs monotherapy of carbamazepine, phenytoin, or valproic acid. Healthy controls (HC) subjects were recruited from car free day event routinely held in Mataram as well as the patient's relatives. The inclusion criteria for the $\mathrm{HC}$ subjects were no history of seizures or epilepsy, aged 18-50 years old and at least junior high school graduate (nine-year-education). The exclusion criteria for both groups include clinically significant depression, prior history for the diagnosis of schizophrenia and dementia, and under treatment of benzodiazepine, antipsychotic and antidepressant at the time of cognitive examination. The existence of clinically significant depression was assessed using Beck Depression Inventory-II (BDI-II) and subjects were considered to have clinically significant depression if BDI-II test showed score of $14-63^{22}$. This study was conducted from September 2017 to March 2019. This study was approved by the Ethical Committee for Medical Research, Universitas Mataram (Register Number 213/UN18.8/ETIK/2017). All subjects provided written informed consent prior to their participation.

\section{Data collection}

The current study collected data on the demographic and clinical characteristics of epilepsy and HC groups, such as age, gender, years of education, Montreal Cognitive Assessment test in Indonesian version (MoCA-Ina) score and scores of cognitive domain listed in MoCA-Ina test, i.e. visuospatial and executive functions, naming, attention, language, abstract thinking, delayed memory, and orientation. The characteristics mentioned above were the continuous variables, excluding gender which was assigned as categorical variable. The categorical variables were the demographic and clinical characteristics taken only from epilepsy group which include age, gender, years of education, age at epilepsy onset, years of treatment, type of seizure, etiology, first-generation AEDs used (carbamazepine, phenytoin, or valproic acid) and cognitive status. Age was determined based on the median value of age and being categorized into younger ( $\leq$ median value of age) and older (> median value of age). With regards to the categorization used in the previous study, years of education was categorized into $<12$ and $\geq 12$ years $^{23}$, age at epilepsy onset into $\leq 18$ and $>18$ years $^{24}$, and years of treatment into $<2$ and $\geq 2$ years $^{25}$. Additionally, type of seizure (focal vs generalized) and etiology (idiopathic vs structural) were categorized based on the previous study as well ${ }^{18}$.

The cognitive status was categorized into cognitive decline and normal based on the grade of MoCA-Ina score. MoCA-Ina is a validated tool for cognitive assessment in the Indonesian as reliability of the instrument has a kappa coefficient of $0.82^{26}$. The MoCA-Ina score is computed as the cumulative scores of its cognitive domains which ranged from $0-30$. Subjects with score of 26-30 were considered as normal, while those with score of $0-25$ were indicative of cognitive decline.

\section{Statistical analysis}

Both demographic and clinical characteristics in epilepsy and HC groups, including age, gender, years of education, MoCA-Ina score, and score of cognitive domains listed in MoCA-Ina test, i.e. visuospatial and executive functions, naming, 
attention, language, abstract thinking, delayed memory and orientation scores were presented as mean $(95 \% \mathrm{Cl})$ unless otherwise stated. Firstly, the analysis was conducted to determine the differences in the mean of age, gender, MoCA-Ina score, and scores of cognitive domains listed in MoCA-Ina test as well as the proportion of gender between epilepsy and $\mathrm{HC}$ groups. The normality test of the continuous variables (means of age, years of education, MoCA-Ina score, and scores of cognitive domain listed in MoCA-Ina test) was performed. Independent t-test was computed to determine the differences in the continuous variables between the epilepsy and $\mathrm{HC}$ groups. Meanwhile, the difference in the proportion of gender (categorical variable) between epilepsy and $\mathrm{HC}$ groups was analyzed using chi-square test.

The second analysis was carried out to determine the association between demographic and clinical characteristics and cognitive decline among epilepsy patients. The association between discrete independent variables, i.e. age (younger vs older), gender (male vs female), years of education ( $<12$ vs $\geq 12$ years), age at epilepsy onset ( $\leq 18$ vs $>18$ years), years of treatment $(<2$ vs $\geq 2$ years), type of seizure (focal vs generalized), and etiology (idiopathic vs structural) and cognitive status (decline vs normal) (dependent variable) among epilepsy patients were examined using simple binary logistic regression analysis. The independent variables showing $p<0.25$ in simple binary logistic regression analysis were then entered into a multiple logistic regression model in which odds ratio (OR) with 95\% confidence interval were reported. The analysis was performed using IBM SPSS 22.0 and statistical significance was set at $\mathrm{p}<0.05$.

\section{RESULTS}

Demographic and clinical characteristics of epilepsy and healthy control subjects

This study recruited 186 subjects (93 epilepsy patients and 93 healthy control subjects). As shown in Table 1, it revealed that there were no significant difference in the means of age and years of education and the distribution of gender between epilepsy and healthy control subjects $(p>0.05)$. Meanwhile, the means of MoCA-Ina, visuospatial and executive functions, naming, attention, language, abstract thinking, delayed memory, and orientation scores in healthy controls were significantly higher compared that of the epilepsy subjects $(p<0.05)$.

Table 1. Demographic and clinical characteristics of epilepsy and healthy control groups

\begin{tabular}{|c|c|c|c|}
\hline \multirow[t]{2}{*}{ Characteristic } & \multicolumn{2}{|c|}{ Mean $(95 \% \mathrm{Cl})$, unless otherwise stated } & \multirow[t]{2}{*}{ p-value } \\
\hline & Epilepsy $(n=93)$ & $\mathrm{HC}(\mathrm{n}=93)$ & \\
\hline Age in years & $29.36(27.43-31.30)$ & $28.99(27.14-30.84)$ & $0.780^{\mathrm{a}}$ \\
\hline \multicolumn{4}{|l|}{ Gender, $\mathrm{n}(\%)$ : } \\
\hline Male & $47(50.5)$ & $54(58.1)$ & \\
\hline Female & $46(49.5)$ & 39 (41.9) & $0.303^{b}$ \\
\hline Years of education & $12.41(11.96-12.86)$ & $12.78(12.31-13.25)$ & $0.261^{\mathrm{a}}$ \\
\hline MoCA-Ina score & $21.69(20.67-22.71)$ & $26.17(26.66-26.69)$ & $<0.001^{c *}$ \\
\hline \multicolumn{4}{|l|}{ Scores of MoCA-Ina components: } \\
\hline Visuospatial/executive function & $3.54(3.27-3.81)$ & $4.35(4.20-4.50)$ & $<0.001^{c *}$ \\
\hline Naming & $2.45(2.29-2.67)$ & $2.94(2.90-2.99)$ & $<0.001^{\mathrm{c} *}$ \\
\hline Attention & $4.61(4.34-4.89)$ & $5.36(5.20-5.53)$ & $<0.001^{\mathrm{c} *}$ \\
\hline Language & $1.98(1.76-2.20)$ & $2.78(2.69-2.88)$ & $<0.001^{\mathrm{c} *}$ \\
\hline Abstract thinking & $1.22(1.06-1.37)$ & $1.38(1.23-1.52)$ & $0.137^{a}$ \\
\hline Delayed memory & $2.09(1.73-2.44)$ & $3.20(2.92-3.49)$ & $<0.001^{c *}$ \\
\hline Orientation & $5.43(5.21-5.65)$ & $5.89(5.83-5.96)$ & $<0.001^{c *}$ \\
\hline
\end{tabular}

$\bar{a}=$ Indendent $t$-test, ${ }^{b}=$ chi-square test, ${ }^{c}=$ Mann-Whitney $U$ test, ${ }^{*}$ Significant difference $(p<0.05)$

$H C=$ healthy controls group; MoCA-Ina=Montreal Cognitive Assessment in Indonesia version.

Demographic and clinical characteristics of epilepsy subjects receiving first-generation AEDs

Ninety-three epilepsy subjects involved in this study were epilepsy outpatients from two main referral hospital in Mataram, Mutiara Sukma Mental Hospital $(n=38)$ and Mataram General Hospital $(n=55)$, respectively. Table 2 summarizes the demographic and clinical characteristics of epilepsy subjects.
Most subjects had high educational level, idiopathic etiology of epilepsy, and took phenytoin as antiepileptic drugs. The structural etiology identified comprised head trauma $(n=16)$, stroke $(n=3)$, and infection/inflammation $(n=2)$. The result of this study showed that prevalence of cognitive decline based on MoCAIna test among epilepsy subjects was high (75.3\%). 
Table 2. Demographic and clinical characteristics of epilepsy subjects

\begin{tabular}{|c|c|}
\hline Characteristics & Frequency (\%) \\
\hline \multicolumn{2}{|l|}{ Age } \\
\hline$\leq 27$ years & $46(49.5)$ \\
\hline$>27$ years & $47(50.5)$ \\
\hline \multicolumn{2}{|l|}{ Gender } \\
\hline Male & 47 (50.5) \\
\hline Female & $46(49.5)$ \\
\hline \multicolumn{2}{|l|}{ Years of education } \\
\hline$<12$ years & $24(25.8)$ \\
\hline$\geq 12$ years & $69(74.2)$ \\
\hline \multicolumn{2}{|l|}{ Age at epilepsy onset } \\
\hline$\leq 18$ years & $46(49.5)$ \\
\hline$>18$ years & $47(50.5)$ \\
\hline \multicolumn{2}{|l|}{ Types of seizure } \\
\hline Focal & $48(51.6)$ \\
\hline Generalized & $45(48.4)$ \\
\hline \multicolumn{2}{|l|}{ Etiology } \\
\hline Idiopathic & $72(77.4)$ \\
\hline Structural & $21(22.6)$ \\
\hline \multicolumn{2}{|l|}{ Years of treatment } \\
\hline$<2$ years & $40(43.0)$ \\
\hline$\geq 2$ years & $53(57.0)$ \\
\hline \multicolumn{2}{|l|}{ First-generation AEDs } \\
\hline Phenytoin & $44(47.3)$ \\
\hline Carbamazepine & $35(37.6)$ \\
\hline Valproic acid & $14(15.1)$ \\
\hline \multicolumn{2}{|l|}{ Cognitive status } \\
\hline Normal (MoCA-Ina score 26-30) & $23(24.7)$ \\
\hline Decline (MoCA-Ina score 0-25) & $70(75.3)$ \\
\hline
\end{tabular}

AEDs=antiepileptic drugs; MoCA-Ina=Montreal Cognitive Assessment in Indonesia version.

Association between characteristics of epilepsy subjects receiving first-generation AEDs and cognitive decline

Table 3 presents the results of simple logistic regression analysis and the final model of multiple logistic regression in examining the association between both demographic and clinical characteristics and cognitive decline among epilepsy subjects. In the final model of

multiple logistic regression, male gender was the only characteristic significantly associated with cognitive decline in epilepsy subjects $(\mathrm{OR}=3.23$, $95 \% \mathrm{Cl}=(1.06-9.88, \mathrm{p}=0.039)$. Nevertheless, age at epilepsy onset, type of seizure, and etiology of epilepsy as the clinical characteristics of epilepsy subjects were not significantly associated with the cognitive decline.

\section{DISCUSSION}

This initial study was aimed at determining the characteristics of cognitive status of epilepsy outpatients receiving monotherapy (phenytoin, carbamazepine, and valproic acid). A comparison to the status of cognitive function between epilepsy patients and healthy subjects was done, followed by contrasting the demographic and clinical characteristics of both subjects. This investigation was carried out using Montreal Cognitive Assessment test in Indonesian version (MoCA-Ina), a cognitive assessment instrument validated for Indonesian population ${ }^{26}$. The findings showed that with comparable means of age and educational level and proportion of gender in both groups (Table 1), the epilepsy patients had lower MoCA-Ina score than the healthy subjects, as well as scores of almost all cognitive domains that composed MoCA-Ina instrument, excluding the abstract thinking domain. The insignificant difference in abstract thinking score between epilepsy patients and healthy subjects is likely related to the equal years of education from both groups. This was due to the reason that abstract thinking is a knowledge-related cognitive process and the integrity of its function is mainly determined by level of education ${ }^{27}$. The lower scores of cognitive domains which construct MoCA-Ina instrument among epilepsy patients in this study, namely visuospatial and executive, naming, attention, language, delayed memory, and orientation functions represent the dysfunction of brain areas carrying these cognitive functions ${ }^{28}$. 
Table 3.Binary logistic regression analysis showing the association between clinical and demographic characteristic and cognitive status among epilepsy subjects

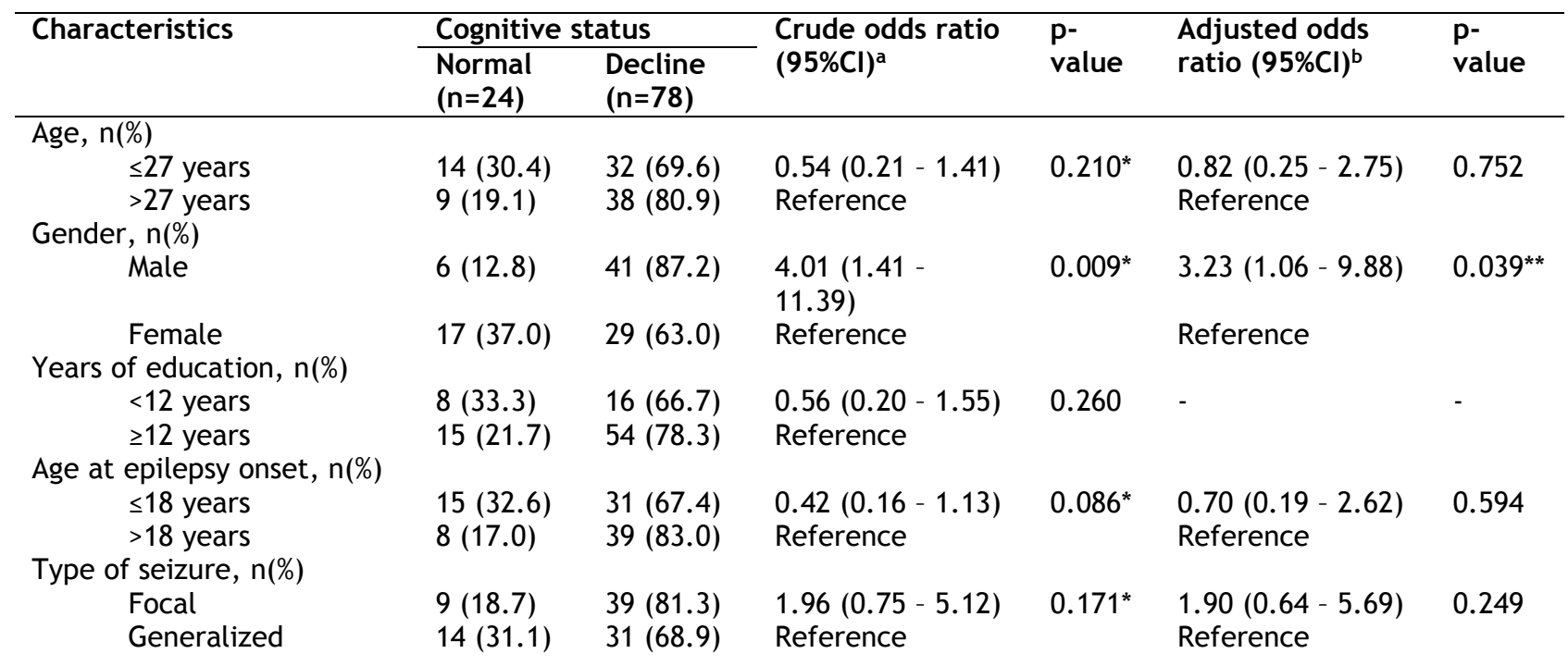

\begin{tabular}{|c|c|c|c|c|c|c|}
\hline \multicolumn{7}{|l|}{ Etiology, n(\%) } \\
\hline Idiopathic & $21(29.2)$ & $51(70.8)$ & $0.26(0.06-1.20)$ & $0.083^{*}$ & $0.42(0.08-2.26)$ & 0.315 \\
\hline Structural & $2(9.5)$ & 19 (90.5) & Reference & & Reference & \\
\hline \multicolumn{7}{|l|}{ Years of treatment, $n(\%)$} \\
\hline$<2$ years & $10(25.0)$ & $30(75.0)$ & $0.98(0.38-2.52)$ & 0.985 & - & - \\
\hline$\geq 2$ years & $13(24.5)$ & 40 (75.5) & Reference & & & \\
\hline \multicolumn{7}{|l|}{ AEDs, $n(\%)$} \\
\hline Carbamazepine & $9(25.7)$ & $26(74.3)$ & $1.30(0.66-2.57)$ & 0.446 & - & - \\
\hline Phenytoin & $8(18.2)$ & $36(81.8)$ & & & & \\
\hline Valproic acid & $6(42.9)$ & $8(57.1)$ & Reference & & & \\
\hline
\end{tabular}

asimple logistic regression analysis; ${ }^{b}$ Multiple logistic regression analysis

*Eligible for multiple logistic regression analysis; ${ }^{*}$ Significant association $(p<0.05)$

Generally, the results of this study were in accordance with the findings from previous studies $^{6,29-31}$. Phabphal and Kanjanasatien assessed cognitive status of epilepsy patients receiving monotherapy and polytherapy antiepileptic drugs (AEDs) using MoCA instrument validated in their respective languages and showed that epilepsy patients with cognitive decline had lower scores of all components compared to those with normal cognitive function ${ }^{29}$. Witt and Helmstaedter reviewed the impact of early stage of epilepsy in adults before medical treatment initiated to their cognitive function and revealed that among all cognitive domains, attention, memory, and executive functions were the most cognitive domain affected in those subjects ${ }^{6}$. Nevertheless, all cognitive domains can be impaired in epilepsy patients and their vulnerability is determined by the demographic and clinical characteristics of the patients.

This study also revealed a high prevalence of cognitive decline among epilepsy patients treated with monotherapy of first-generation antiepileptic drugs with comparable age, gender, age at epilepsy onset, type of seizure, and years of treatment. The high proportion of epilepsy patients with high education is likely to be due to the recruitment of the subjects assigned to those who had at least junior high school graduate (nine-year-education). This strengthened the result from previous study that overall, epilepsy with idiopathic etiology had high prevalence and incidence and it was considered to have a genetic basis $^{31}$. Most of the epilepsy patients received phenytoin (47.3\%), followed by carbamazepine (37.6\%) and valproic acid (15.1\%). The high prevalence of cognitive decline in these subjects can be explained through theoretical approach, where in the condition of epilepsy, epileptic seizures as well as interictal epileptiform discharge can induce neuronal damage in particular brain areas carrying particular cognitive functions ${ }^{28}$. This condition will decrease the cognitive status through the mechanism of modulation of cognitive processes and long-term structural changes. As a result, this will get inhibitory effect on impulse produced by cognitive processes as well ${ }^{13}$.

In most cases, the vulnerability of epilepsy patients suffering from epilepsy-associated cognitive decline is determined by demographic and clinical characteristics of the patients and characteristics of the treatment. This study implied that among those characteristics, gender was the only variable significantly associated with the prevalence of cognitive decline among these subjects (Table 3 ). A male epilepsy patient has 
greater risk for cognitive decline than female. Female epilepsy patient is protective against cognitive decline due to the role of high level of estrogen in female of reproductive age. Estrogen is considered presenting an important role in many cognitive processes related to knowledge in the brain, including attention, learning and memory consolidation, abstract thinking and language production ${ }^{32}$. Theoretically, by binding to its receptors in many parts of brain involved in cognitive functioning, i.e. prefrontal cortex, hippocampus, basal forebrain, and striatum, estrogen, it will induce the increase of antioxidants which subsequently decrease the oxidative stress ${ }^{33}$, the damaging process involved in epileptic seizure and IEDs-induced neuronal death ${ }^{28}$.

In contrast, the characteristics of age, years of education, age at epilepsy onset, type of seizure, and etiology of epilepsy did not associate significantly with the prevalence of cognitive decline among these epilepsy subjects in multiple logistic regression model (Table 3 ). This findings was on the contrary to the results of several reviews showing that interaction of these variables will affect the cognitive decline in epilepsy patients ${ }^{11,30}$.

Due to the complexity of the disease, the previous studies assessing cognitive status using MoCA instrument and identifying variables associated with the prevalence of cognitive decline in different population of epilepsy patients also revealed varied results. Wang et al. analyzed variables for cognitive decline in epilepsy patients using multiple linear regression analysis and showed that high level of education, good control of seizure, receiving monotherapy of AEDs, and absent of depression were protective variables against cognitive decline in these subjects ${ }^{21}$. Gavrilovic et al. examined correlation between demographic and clinical characteristics and cognitive decline among drug-resistant and drugsensitive epilepsy patients in a cohort study and showed that duration of epilepsy, control of seizure, and EEG findings are significant predictors for cognitive decline in these subjects ${ }^{34}$. These different results from the prior studies presented previously are more likely related to different in methods and study design used as well as population of epilepsy patients observed.

In addition, this study also exposed that firstgeneration of AEDs, namely carbamazepine, phenytoin, and valproic acid, used as monotherapy did not associate with the prevalence of cognitive decline in epilepsy patients. This result might be due to the equality of side effect profiles on cognitive status of these three $A E D s^{17}$. Unfortunately, data comparing the effects of monotherapy of these three AEDs on the cognitive status of epilepsy patients remain scarce. Witt and Helmstaedter suggested in their opinion that the impact of AEDs on cognitive function varies from subjects of epilepsy, in terms of cognitive domains affected and the severity as well as the reversibility of the cognitive impairment ${ }^{35}$. Desai reviewed the effect of AEDs on cognition and showed that first-generation AEDs, including carbamazepine, phenytoin, and valproic acid, had more pronounced effects on cognitive status compared with those newgeneration ones ${ }^{36}$. On the other hand, due to their large availability, affordable price, proven efficacy and well-known adverse effects of the first-generation AEDs, these drugs are still used broadly, especially in developing countries, including Indonesia ${ }^{3,37}$. Eddy et al. reviewed the cognitive impact of antiepileptic drugs and confirmed that first-generation AEDs however did not merely show negative effects on cognitive status, but also being reported to provide improvements to cognitive performance in epilepsy patients ${ }^{16}$. Further study comparing the cognitive impacts of first-generation and newgeneration AEDs in large population of epilepsy patients is required, since the results of this study will affect health policy making by local health authorities in developing countries in the provision of AEDs for epilepsy patients.

The present study has some limitations. Since the sample of epilepsy patients were from those having a minimum of 9-year-education, the results of this research cannot be generalized in all population of epilepsy patients. This is particularly for the epilepsy patients with lower level of education and illiterate, living in rural region of developing countries including Indonesia. Conversely, this minimum year of education is beneficial in reducing recall bias of information as well as bias in MoCA-Ina test results. This study did not consider bipolar affective disorders as a confounding factor for cognitive impairment, hence the effect of these disorders on cognitive performance among epilepsy patients cannot be ruled out. Due to the lack of data about prevalence of epilepsyassociated cognitive decline in the population of Indonesian epilepsy patient, this information becomes valuable as a basis for health policy making by local health authority for epilepsyassociated cognitive decline intervention as well as AEDs provision.

\section{CONCLUSION}

In conclusion, the cognitive status of epilepsy patients using monotherapy with carbamazepine, phenytoin, and valproic acid were decreased significantly compared with healthy subjects. The prevalence of cognitive decline among these epilepsy patients is high and gender is the only characteristic of epilepsy patients significantly associated with this cognitive decline. Epilepsy patients with male gender have greater risk for cognitive decline. 


\section{REFERENCES}

1. Fiest KM, Sauro KM, Wiebe S, Patten SB, Kwon C-S, Dykeman J, et al. Prevalence and incidence of epilepsy A systematic review and meta-analysis of international studies. Neurology. 2017;88:296-303. Available from: doi: 10.1212/WNL.0000000000003509

2. Beghi E, Giussani G. Aging and the Epidemiology of Epilepsy. Neuroepidemiology. 2018;51:216-23. Available from: doi: 10.1159/000493484

3. Mac TL, Tran D, Quet F, Odermatt P, Preux $\mathrm{P}$, Tan CT. Epidemiology, aetiology, and clinical management of epilepsy in Asia: a systematic review. Lancet Neurol. 2007;6:533-43. Available from: doi: $10.1016 /$ S1474-4422(07)70127-8

4. Trinka E, Kwan P, Lee B, Dash A. Epilepsy in Asia: Disease burden, management barriers, and challenges. Epilepsia. 2019;60:7-21.

5. Nau A, Mwape K, Wiefek J, Schmidt K, Abatih E, Dorny $P$, et al. Cognitive impairment and quality of life of people with epilepsy and neurocysticercosis in Zambia. Epilepsy Behav. 2018;80:354-9. Available from:

doi: 10.1016/j.yebeh.2017.10.042

6. Witt J, Helmstaedter C. Cognition in the early stages of adult epilepsy. Seizure. 2015;26:65-8. Available from: http://dx.doi.org/10.1016/j.seizure.2015 .01 .018

7. Witt J, Helmstaedter C. Should cognition be screened in new-onset epilepsies ? A study in 247 untreated patients. J Neurol. 2012;259:1727-31. Available from: DOI $10.1007 / \mathrm{s} 00415-012-6526-2$

8. Leeman-Markowski BA, Schachter SC. Cognitive and behavioral interventions in epilepsy. Curr Neurol Neurosci Rep. 2017;17(5):42.

9. Szemere E, Jokeit H. Quality of life is social - Towards an improvement of social abilities in patients with epilepsy. Seizure. 2015;26:12-21. Available from: http://dx.doi.org/10.1016/j.seizure.2014 .12 .008

10. Jost J, Millogo A, Preux P-M. Antiepileptic Treatments in Developing Countries. Curr Pharmacautical Des. 2017;23:1-9.

11. Lodhi S, Agrawal N. Neurocognitive problems in epilepsy. Adv Psychiatr Treat.
2012;18:232-40.

12. Salassie A, Wannamaker B, Smith G, Wagner J, Edwards J, Martz G, et al. Risk Factors of Epilepsy Outcomes: Comorbidities in Population with Epilepsy. South Carolina; 2014.

13. Lenck-Santini P-P, Scott RC. Mechanisms Responsible for Cognitive Impairment in Epilepsy. Cold Spring Harb Perspect Med. 2015;5:a022772.

14. Landi S, Petrucco L, Sicca F, Ratto GM. Transient Cognitive Impairment in Epilepsy. Front Mol Neurosci. 2019;11:458.

15. Witt J, Helmstaedter C. Monitoring the cognitive effects of antiepileptic pharmacotherapy - approaching the individual patient. Epilepsy Behav. 2013;26:450-6. Available from: http://dx.doi.org/10.1016/j.yebeh.2012. 09.015

16. Eddy CM, Rickards HE, Cavanna AE. The cognitive impact of antiepileptic drugs. Ther Adv Neurol Disord. 2011;4(6):385407. Available from: doi: $10.1177 / \% 0 A 1756285611417920$

17. Park S-P, Kwon S-H. Cognitive Effects of Antiepileptic Drugs. J Clin Neurol. 2008;4:99-106. Available from: 10.3988/jcn.2008.4.3.99

18. Budikayanti A, Qadri LM, Syeban Z, Indrawati LA, Octaviana F. Adverse Events of Antiepileptic Drugs Using Indonesian Version of Liverpool Adverse Events Profile. Neurol Res Int. 2018;2018:8490639. Available from: doi: $10.1016 /$ S1474-4422(07)70127-8

19. Harahap $\mathrm{H}$, Indrayana $\mathrm{Y}$, Amalia $\mathrm{E}$. Treatment Pattern and Cognitive Function in Epilepsy Patients in Mutiara Sukma Mental Hospital. J Kedokt Brawijaya. 2017;29(04):335-40. Available from: doi: http://dx.doi.org/10.21776/ub.jkb.2017. 029.04.9

20. Loring DW, Marino S, Meador KJ. Neuropsychological and Behavioral Effects of Antiepilepsy Drugs. Neuropsychol Rev. 2007;17:413-25.

21. Wang L, Chen S, Liu C, Lin W, Huang $\mathrm{H}$. Factors for cognitive impairment in adult epileptic patients. Brain Behav. 2020;10:e01475.

22. Wang Y-P, Gorenstein C. Assessment of depression in medical patients : A systematic review of the utility of the Beck Depression Inventory-II. Clinics. 
2013;68(9):1274-87.

23. Crowe M, Clay OJ, Martin RC, Howard VJ, Wadley VG, Sawyer P, et al. Indicators of Childhood Quality of Education in Relation to Cognitive Function in Older Adulthood. J Gerontol A Biol Sci Med Sci. 2013;68(2):198-204.

24. Bicalho MAH, Sukys-Claudino L, Guarnieri R, Lin K, Walz R. Socio-demographic and clinical characteristics of Brazilian patients with epilepsy who drive and their association with traffic accidents. Epilepsy Behav. 2012;24(2):216-20. Available from: http://dx.doi.org/10.1016/j.yebeh.2012. 04.114

25. Hixson JD. Stopping Antiepileptic Drugs: When and Why ? Curr Treat Options Neurol. 2010;12:434-42.

26. Husein $\mathrm{N}$, Lumempouw $\mathrm{S}$, Ramli $\mathrm{Y}$, Herqutanto. Montreal Cognitive Assessment Versi Indonesia (MOCA-INA) Untuk Skrining Gangguan Fungsi Kognitif. Neurona. 2010;27(4):15-22.

27. Dumontheil I. Development of abstract thinking during childhood and adolescence: The role of rostrolateral prefrontal cortex. Dev Cogn Neurosci. 2014;10:57-76. Available from: http://dx.doi.org/10.1016/j.dcn.2014.07. 009

28. Lorigados L, Orozco S, Morales L, Estupinan B, García I, Rocha L. Excitotoxicity and neuronal death in epilepsy. Biotecnol Apl. 2013;30:9-16.

29. Phabphal K, Kanjanasatien J. Montreal Cognitive Assessment in cryptogenic epilepsy patients with normal Mini-Mental State Examination. Epileptic Disord. 2011;13(4):375-81.

30. Mula M. Cognitive dysfunction in patients with epilepsy: focus on clinical variables. Future Neurol. 2015;10(1):41-8.

31. Banerjee PN, Filippi D, Hauser WA. The descriptive epidemiology of epilepsy-a review. Epilepsy Res. 2009;85(1):31-45.

32. Luine VN. Estradiol and cognitive function: Past, present and future. Horm Behav. 2014;66(4):602-18.

33. Rahman $\mathrm{A}$, Jackson $\mathrm{H}$, Hristov $\mathrm{H}$, Isaacson RS, Saif N, Shetty T, et al. Sex and Gender Driven Modifiers of Alzheimer's: The Role for Estrogenic Control Across Age, Race, Medical, and Lifestyle Risks. Front Aging Neurosci. 2019;11:315.

34. Gavrilovic A, Toncev G, Matic TB, Vesic K, Zivojinovic IJ, Gavrilovic J. Impact of epilepsy duration, seizure control and EEG abnormalities on cognitive impairment in drug-resistant epilepsy patients. Acta Neurol Belg. 2019;119(3):403-10. Available from: http://dx.doi.org/10.1007/s13760019-01090-x

35. Witt J-A, Helmstaedter C. How can we overcome neuropsychological adverse effects of antiepileptic drugs? Expert Opin Pharmacother. $\quad 2017 ; 18(6): 551-4$. Available from: http://dx.doi.org/10.1080/14656566.201 7.1309025

36. Desai JD. Epilepsy and cognition. J Pediatr Neurosci. 2008;3:16-29.

37. Cameron A, Bansal A, Dua T, Hill SR, Moshe SL, Mantel-teeuwisse AK, et al. Mapping the availability, price, and affordability of antiepileptic drugs in 46 countries. Epilepsia. 2012;53(6):962-9. 